\title{
Condições de trabalho e saúde de professores pré-escolares da rede pública de ensino de Pelotas, RS, Brasil
}

\author{
Working and health conditions of preschool teachers of the public \\ school network of Pelotas, State of Rio Grande do Sul, Brazil
}

Luciane Goulart da Silva ${ }^{1}$

Marcelo Cozzensa da Silva ${ }^{1}$

${ }^{1}$ Programa de Pós-

Graduação em Educação

Física, Escola Superior de Educação Física,

Universidade Federal de

Pelotas. R. Luiz de Camões

625. 96.055-630, Pelotas RS Brasil.

lucianegoularts@yahoo.com.br
Abstract This study describes the working and health conditions of preschool teachers from the public school network in Pelotas, State of Rio Grande do Sul. A descriptive census was conducted in schools of the city and the state that offered preschool classes. The questionnaire included social and demographic, behavioral, nutritional, health and work issues. All teachers were female, more than 55\% were classified as being overweight, $12.6 \%$ were smokers and $73 \%$ were not sufficiently physically active during their leisure time. With respect to the working conditions, $66.7 \%$ reported working in an uncomfortable posture, $40.5 \%$ considered the desks and furniture inadequate, 50.5\% replied that the intervals between classes and activities are insufficient for resting. The prevalence of back, thoracic, neck and shoulder pain was high, and $17.8 \%$ tested positive for minor psychiatric disorders. The prevalence rates for occupational exposure and poor health conditions of preschool teachers are significant and can interfere in the quality of life and work of these individuals.

Key words Working conditions, Occupational health, Occupational risks, Teachers, School health, Epidemiology
Resumo O presente estudo descreve as condições de trabalho e saúde de professores pré-escolares da rede pública de ensino da cidade de Pelotas, RS. Foi realizado um estudo descritivo do tipo censo nas escolas do município e estado que ofereciam a pré-escola. Foi aplicado um questionário incluindo questões sociodemográficas, econômicas, comportamentais, nutricionais, de saúde e trabalho. Todos os professores estudados eram do sexo feminino, mais de 55\% foram classificadas com sobrepeso, 12,6\% fumavam atualmente e 73,0\% eram insuficientemente ativos no tempo de lazer. Quanto às condições de trabalho, 66,7\% relataram trabalhar em posição incômoda, 40,5\% consideraram o mobiliário de trabalho inadequado e $50,5 \%$ responderam que as pausas realizadas $d u$ rante o trabalho são insuficientes para o descanso. A prevalência de dor nas regiões lombar, torácica, pescoço e ombros foi elevada e 17,8\% das entrevistadas apresentou positividade para transtornos psiquiátricos menores. As prevalências encontradas para as exposições ocupacionais e as más condições de saúde dos professores pré-escolares são importantes e podem interferir na qualidade de vida e de trabalho desses indivíduos.

Palavras-chave Condições de trabalho, Saúde do trabalhador, Riscos ocupacionais, Docentes, Saúde escolar, Epidemiologia 


\section{Introdução}

A Educação Infantil, primeira etapa da educação básica, assume cada vez mais destaque na discussão da educação brasileira. A Lei de Diretrizes e Bases (LDB) ${ }^{1}$, de 20 de dezembro de 1996, estabelece as diretrizes e bases da educação nacional, tratando na sua Seção II, artigos 29, 30 e 31, especificamente da educação infantil, e, pela primeira vez na história das legislações brasileiras proclamou a educação infantil como direito das crianças de 0 a 6 anos e dever do Estado.

Recentes medidas legais modificaram o atendimento às crianças da educação infantil, pois alunos com seis anos de idade devem obrigatoriamente estar matriculados no primeiro ano do Ensino Fundamental. O Projeto de Lei no 144/2005², aprovado pelo Senado em 25 de janeiro de 2006, estabelece a duração mínima de nove (9) anos para o ensino fundamental, com matrícula obrigatória a partir dos seis (6) anos de idade. Essa medida visava à implantação da referida lei pelos Municípios, Estados e Distrito Federal até 2010. A nomenclatura para a Educação Infantil estabeleceu-se da seguinte maneira: Educação Infantil: até cinco (5) anos de idade; Creche: até três (3) anos de idade; Pré-escola: quatro a cinco (4 a 5) anos de idade.

Ensinar é, em geral, uma atividade altamente estressante, com repercussões evidentes na saúde física, mental e no próprio desempenho profissional dos professores ${ }^{3}$. Segundo Mendes ${ }^{4}$ e Chong e Chan ${ }^{5}$, além de ministrar aulas, os professores devem realizar trabalhos administrativos, planejar, reciclar-se, investigar, orientar alunos e interagir com os pais de seus alunos. Além disso, organizar atividades extracurriculares, participar de reuniões de coordenação, seminários, conselhos de classe; preencher relatórios relativos às dificuldades de aprendizagem e, muitas vezes, cuidar do patrimônio, materiais, recreios e locais de refeições fazem parte de sua rotina de trabalho. Essa variedade e acúmulo de funções de trabalho podem levar os professores a problemas de saúde mental e física ${ }^{5}$.

Paschoal e Machado ${ }^{6}$ relatam que o cansaço físico das professoras que atuam na educação infantil é ainda mais acentuado porque o trabalho com crianças menores exige muita desenvoltura física e equilíbrio emocional. Diferentemente da performance em outras séries do ensino, o professor de pré-escola despende um maior contato físico no trabalho com seus alunos, realizando tarefas específicas como carregar crianças no colo, levantá-las do chão, agachar, ajoelhar e curvar-se para acompanhar as atividades esco- lares dos mesmos. Como consequência dessa sobrecarga laboral os professores estão sujeitos a uma série de problemas que vão desde os transtornos psiquiátricos como tensão, ansiedade, frustração e depressão até os problemas relacionados à saúde musculoesquelética.

No Brasil existe uma escassez de estudos sobre condições de trabalho e saúde do professor pré-escolar em comparação com trabalhadores de outras profissões ${ }^{7}$. Dois importantes estudos nessa área foram feitos por $\mathrm{Codo}^{8}$, enfocando saúde mental de professores do ensino fundamental e médio e Silvany-Neto et al. ${ }^{9}$ envolvendo processo e condições de trabalho e as repercussões sobre a saúde do educador. Conhecer as condições de trabalho e de saúde desses professores é de grande importância no sentido de fornecer informações que possam ser úteis no auxílio aos órgãos promotores de políticas públicas a criarem medidas que contemplem melhorias, tanto no ambiente de trabalho como para a saúde dessa população, colaborando assim para a melhoria da qualidade de ensino.

Portanto, o objetivo do presente estudo foi descrever as condições de trabalho e saúde de professores pré-escolares da rede pública de ensino da cidade de Pelotas, Rio Grande do Sul.

\section{Métodos}

Realizou-se um estudo epidemiológico de natureza descritiva, sobre as condições de trabalho e saúde dos professores pré-escolares da rede municipal e estadual de ensino na cidade de Pelotas (RS). O Protocolo do estudo foi aprovado pelo Comitê de Ética em Pesquisas da Escola Superior de Educação Física da Universidade Federal de Pelotas.

A rede pública municipal e estadual é constituída por um total de cento e quarenta e três (143) escolas, das quais apenas sessenta e duas (62) oferecem Educação Infantil, com 116 professores pré-escolares. Foram pesquisadas professoras de Educação Infantil - pré-escolar que atendem crianças de três a cinco ( 3 a 5 ) anos de idade distribuídas pelas 62 escolas da rede pública, sendo que quatro professoras estaduais foram selecionadas por sorteio para participar do Grupo Focal. Uma professora se encontrava afastada por motivo de doença e não houve nenhuma recusa por parte das educadoras, totalizando, assim, cento e onze (111) entrevistadas.

Como critério de inclusão considerou-se todos os professores pré-escolares de escolas públi- 
cas efetivos da zona urbana da cidade de Pelotas, ativos no período da coleta de dados e trabalhando em sala de aula, diretamente com os alunos. Foram excluídas as professoras que estavam em algum tipo de afastamento ou licença no período de recolhimento de dados, e aquelas que participaram do Grupo Focal (GF) realizado para auxiliar na construção do instrumento final.

Para realizar as entrevistas foi solicitada autorização junto à Secretaria Municipal de Educação e à $5^{\mathrm{a}}$ Coordenadoria Regional de Educação. De posse das respectivas licenças e da lista com os nomes das escolas de Educação Infantil, fez-se contato com a direção das mesmas, para agendar as entrevistas com as professoras pré-escolares, preferencialmente no local de trabalho e de maneira a não atrapalhar as atividades das escolas.

As entrevistas foram realizadas por profissionais treinadas. Antes disso, as professoras assinaram o termo de consentimento livre e esclarecido, quando foram informadas sobre os objetivos da pesquisa e elucidadas quanto ao sigilo mantido sobre as informações dadas, utilizadas somente para fins de pesquisa.

A coleta de dados foi realizada, no período de setembro a novembro de 2010 , por 13 entrevistadoras previamente selecionadas, que passaram por um treinamento de 20 horas. Para garantir maior padronização, além do treinamento, foi utilizado um manual de instruções básicas para orientar os procedimentos durante as entrevistas.

Para um total de $10 \%$ das professoras já entrevistadas foi aplicada uma versão resumida do questionário, incluindo, porém, questões essenciais do instrumento para fins de controle de qualidade.

$\mathrm{O}$ instrumento utilizado para a coleta de dados foi um questionário elaborado pelos autores. O mesmo constituiu-se de 161 questões envolvendo aspectos socioeconômicos (nível econômico - através do questionário $\mathrm{ABEP}$, renda em salários mínimos, escolaridade - anos completos de estudo) demográficos (sexo - masculino e feminino, idade - anos completos, cor da pele - autorrelatada pelo entrevistado) condições de trabalho (horas-aula semanais, turnos de trabalho, número de alunos por turma, pausas durante o período de trabalho, adequação de mobiliário, de iluminação; movimentos repetitivos, esforço físico despendido para levantar e mover objetos pesados, crianças; exposição a ruídos internos e externos em sala-de-aula, ao pó de giz), além de comportamentais em relação à saúde (autopercepção de saúde, hábitos de fumar, nível de atividade física, índice de massa corporal, transtornos psiquiátricos menores e problemas musculoesqueléticos).

As questões referentes às condições de trabalho dos professores foram baseadas em diferentes questionários utilizados em estudos de avaliação desse tema em trabalhadores da educação. Além disso, utilizou-se uma técnica conhecida como GF, técnica de discussão não diretiva em grupo, que reúne pessoas com alguma característica ou experiência comum para discussão de um tema ou área de interesse. O GF foi conduzido com professoras de pré-escolares de uma escola pública para auxiliar na elaboração do questionário. As questões foram pré-testadas em professores de escolas públicas de educação infantil de um município vizinho a Pelotas. Ao final dos processos, o presente questionário ficou com 23 questões fechadas e abertas referentes às condições de trabalho (Jornada de trabalho - horas aula; Número de alunos por turma; falta de materiais - não, sim qual?; Conservação de materiais de trabalho - não, sim; Considera mobiliário de trabalho adequado - não, sim; Como considera iluminação da sala - muito boa, boa, regular, ruim; Distância da residência ao trabalho em Km; Modo de transporte para o trabalho; Possui pausas na jornada de trabalho - não: quantas horas leciona sem pausa, sim: quanto tempo?; Considera pausa suficiente - não, sim; Realiza movimentos repetitivos - não, sim:quais?; Fica em posição incômoda ou viciosa - não, sim: qual?: agachado, em pé, sentado, ajoelhado; Levanta ou carrega peso no trabalho - não, sim: quanto em média em kg?; Possui autonomia para tomada de decisões em aula - não, sim; Sente prejudicado por barulhos, ruídos internos ou externos - não, sim: qual?; Trabalha com ajuda de auxiliar - não, sim; Sente-se valorizado por direção e colegas pelo trabalho que realiza - não, sim; Precisa preencher pareceres fora do horário - não, sim; Existe cobrança dos pais - não, sim: que tipo?; Como considera a satisfação com o trabalho - muito, satisfeito, pouco, insatisfeito; Sente disposição para interagir com a família após o trabalho - não, sim; Deseja abandonar a profissão - não, sim).

O instrumento utilizado para avaliar o nível de atividade física foi o Questionário Internacional de Atividade Física (IPAQ), versão longa, proposto pela Organização Mundial de Saúde (OMS) e pelo Centro de Controle e Prevenção de Doenças (CDC), composto por 27 perguntas que medem a prática de atividade física em quatro domínios: no trabalho, no deslocamento, nas atividades domésticas e no lazer. Para o presente es- 
tudo foi considerado o total de atividade nos domínios do lazer e do trabalho em uma semana habitual e avaliadas as práticas de caminhada, outras atividades físicas moderadas (aquelas que fazem aumentar um pouco os batimentos cardíacos e aceleram um pouco a respiração) e vigorosas (aquelas que fazem aumentar muito os batimentos cardíacos e aceleram muito a respiração). Os indivíduos que relataram a prática semanal igual ou superior a 150 minutos foram considerados ativos. Para construção deste escore, o tempo gasto com a prática de caminhada, atividades físicas moderadas e vigorosas foram somados, sendo que o tempo das atividades vigorosas foi multiplicado por dois. Esta classificação vai ao encontro das recomendações atuais de atividade física ${ }^{10}$.

Os transtornos psiquiátricos menores foram identificados por meio do Self-Report Questionnaire (SRQ-20). O SRQ é recomendado pela Organização Mundial de Saúde para identificar doenças psíquicas comuns, testado e validado por Harding et al. ${ }^{11}$ e validado no Brasil por Mari e Willians ${ }^{12}$. Os Escores de 8 a 20 foram considerados sugestivos de transtornos psiquiátricos menores para o sexo feminino, acompanhando o melhor ponto de corte segundo Mari e Williams ${ }^{12}$.

Para investigar as questões relativas aos sintomas musculoesqueléticos foi utilizado o Questionário Nórdico de Sintomas Musculoesquelé$\operatorname{ticos}^{13}$. Este instrumento foi adaptado culturalmente para a língua portuguesa por Barros e Alexandre ${ }^{14}$ em 2003 e vem sendo amplamente utilizado em estudos epidemiológicos.

O nível socioeconômico foi definido a partir do Critério de Classificação Econômica Brasil que estima o poder de compra das pessoas e famílias urbanas $^{15}$.

O índice de massa corporal (IMC) dos indivíduos foi calculado pelo peso $(\mathrm{Kg})$ referido, dividido pela altura $(\mathrm{cm})$ referida elevada ao quadrado, conforme recomendações da Organização Mundial da Saúde (World Health Organization - WHO ${ }^{16}$.

O banco de dados foi construído no programa Epi Info 6.0, sendo realizada dupla digitação de cada questionário com posterior análise de consistência. Para a análise utilizou-se o programa STATA 10.0. Realizou-se uma análise descritiva dos dados, através do uso de tabelas de frequência para variáveis categóricas e estatísticas descritivas (média e desvio-padrão) para as variáveis numéricas.

\section{Resultados}

Participaram da pesquisa todos os professores (100\%) que trabalhavam no pré-escolar das escolas do estado e município da cidade de Pelotas (RS) em 2010. A média de idade dos entrevistados foi de 39,6 anos ( $d p=8,7$ ), sendo a totalidade do sexo feminino. Observou-se que mais de $84 \%$ das professoras era de cor branca, $63,9 \%$ era casada/vivia com companheiro e $72,1 \%$ tinham filhos. Em relação à escolaridade, a média de anos de estudos encontrada foi de 15,8 (dp = 2,2 ), sendo que $13,5 \%$ possuíam nível médio completo, $39,6 \%$ nível superior e $46,9 \%$ pós-graduação (Tabela 1).

Quanto à renda mensal referente à função docente, constatou-se que $89,2 \%$ recebem entre um e três salários-mínimos e mais de $3 / 4$ das professoras pertencem à classe econômica $\mathrm{B}$. No

Tabela 1. Frequência absoluta (n) e relativa (\%) das variáveis socioeconômicos e demográficos de professoras da rede pública de ensino da zona urbana da cidade de Pelotas (RS), $2010(\mathrm{n}=111)$

\begin{tabular}{lcc}
\hline \multicolumn{1}{c}{ Variáveis } & N & $\%$ \\
\hline Idade (anos) & & \\
$20-29$ & 14 & 12,6 \\
$30-39$ & 39 & 35,1 \\
$40-49$ & 45 & 40,5 \\
50 ou mais & 13 & 11,8 \\
Cor da pele & & \\
Branca & 94 & 84,7 \\
Não branca & 17 & 15,3 \\
Estado civil & & \\
Casada/vive companheiro & 71 & 63,9 \\
Solteira & 40 & 36,1 \\
Escolaridade & & \\
Ensino médio & 15 & 13,5 \\
Superior & 96 & 86,5 \\
Pós-graduação & 52 & 46,9 \\
Nível econômico & & \\
A & 14 & 12,6 \\
B & 86 & 77,4 \\
C & 11 & 10,0 \\
Renda mensal docente & & \\
Até um salário mínimo & 4 & 3,6 \\
Entre um e dois salários & 58 & 52,2 \\
Entre dois e três salários & 41 & 37,0 \\
Mais de 3 salários & 8 & 7,2 \\
Outra atividade remunerada & & \\
Não & 103 & 92,8 \\
Sim & & 7,2 \\
\hline & & \\
\hline & &
\end{tabular}


que se refere à realização de outra atividade remunerada, $7,2 \%$ relataram trabalhar em outra função diferente da docência.

Quanto à rede de ensino, 97,3\% lecionavam na rede municipal e $2,7 \%$ na estadual. Em média estas professoras trabalham na pré-escola há 8,9 anos $(\mathrm{dp}=6,8)$ e sua média de horas-aula semanais perfaz 35,8 horas $(\mathrm{dp}=8,7)$. Mais de $65,0 \%$ das professoras trabalham em uma escola somente, sendo que $60,4 \%(n=67)$ realizam suas atividades nos turnos da manhã e tarde. A média de alunos por sala de aula foi de $16,4(\mathrm{dp}=4,0)$.

A falta de materiais em sala de aula foi referida por $50,4 \%$ das professoras entrevistadas: materiais para educação artística $(37,5 \%)$, brinquedos $(28,6 \%)$, folhas de ofício $(25,0 \%)$ e jogos $(21,4 \%)$.

$\mathrm{Na}$ questão sobre condições de conservação de materiais na sala de aula, 32,4\% responderam haver problemas. Quanto ao mobiliário usado durante a jornada de trabalho, 40,5\% consideraram inadequado (Tabela 2). Sobre as pausas re-

Tabela 2. Frequência absoluta (n) e relativa (\%) das características do trabalho dos professores préescolares das escolas públicas da cidade de Pelotas/ RS, 2010. $(\mathrm{n}=111)$

\begin{tabular}{|c|c|c|}
\hline Variáveis & $\mathbf{N}$ & $\%$ \\
\hline \multicolumn{3}{|c|}{ Más condições materiais } \\
\hline Não & 75 & 67,6 \\
\hline Sim & 36 & 32,4 \\
\hline \multicolumn{3}{|l|}{ Mobiliário } \\
\hline Adequado & 66 & 59,5 \\
\hline Inadequado & 45 & 40,5 \\
\hline \multicolumn{3}{|c|}{ Pausas durante o trabalho } \\
\hline Não & 14 & 12,6 \\
\hline Sim & 97 & 87,4 \\
\hline \multicolumn{3}{|c|}{ Movimentos repetitivos } \\
\hline Não & 89 & 80,2 \\
\hline Sim & 22 & 19,8 \\
\hline \multicolumn{3}{|l|}{ Posições incômodas } \\
\hline Não & 37 & 33,3 \\
\hline Sim & 74 & 66,7 \\
\hline \multicolumn{3}{|l|}{ Carregar peso } \\
\hline Não & 56 & 50,5 \\
\hline Sim & 55 & 49,5 \\
\hline \multicolumn{3}{|l|}{ Barulhos ou ruídos } \\
\hline Não & 51 & 45,9 \\
\hline Sim & 60 & 54,1 \\
\hline \multicolumn{3}{|l|}{ Auxiliar } \\
\hline Não & 58 & 52,3 \\
\hline Sim & 53 & 47,7 \\
\hline
\end{tabular}

alizadas durante o trabalho na pré-escola, 50,5\% das professoras consideraram insuficientes para o descanso.

Entre as professoras estudadas, mais de $19,0 \%$ relataram realizar movimentos repetitivos durante o turno de trabalho e $66,7 \%$ relataram a permanência em posições incômodas ou viciosas frequentemente, sendo as posições agachada $(78,4 \%)$ e em pé $(70,3 \%)$ as mais citadas. Quase metade das entrevistadas referiu carregar peso no trabalho, sendo em média de $11,3 \mathrm{Kg}$ $(\mathrm{dp}=7,9)$. Mais da metade $(54,1 \%)$ considerava que barulhos/ruídos internos ou externos à sala de aula prejudicavam seu trabalho, apontando sobretudo os barulhos dentro da sala de aula $(28,3 \%)$, trânsito $(26,7 \%)$ e crianças no corredor $(23,3 \%)$. A presença de auxiliares para ajudar nas atividades de ensino ocorreu em 47,7\% dos relatos (Tabela 2). Quanto à valorização profissional, aproximadamente $20,0 \%$ não se sentem valorizadas pela direção e por colegas de outras séries com o trabalho desenvolvido na pré-escola; 21,6\% referiram estar pouco satisfeitas com o trabalho e 19,8\% relataram desejo de abandonar a profissão.

A Tabela 3 descreve as variáveis relacionadas às condições de comportamento e saúde das professoras pré-escolares. Em relação à autopercepção de saúde e hábito de fumar, verificou-se que $76,6 \%$ das professoras consideraram sua saúde como sendo muito boa ou boa e $12,6 \%$ admitiram fumar. A mediana de prática semanal de atividade física (atividade física nos domínios de lazer e trabalho) foi de 150 minutos, sendo que 47,8\% não atingiam o mínimo de atividade física recomendada para promoção da saúde (150 min. por semana). Quando analisada somente a prática de atividade física no domínio de lazer, 73,0\% das professoras não atingiram a quantidade mínima recomendada pelos órgãos de saúde. A média de estatura e massa corporal das professoras foi, respectivamente, de $161,3 \mathrm{~cm}(\mathrm{dp}=7,0)$ e 67,1 $\mathrm{Kg}(\mathrm{dp}=11,7)$, consideradas, de acordo com a classificação do índice de massa corporal (IMC), nas categorias referentes a sobrepeso e obesidade, percentual relativo a $55,1 \%$ das entrevistadas.

A prevalência de transtornos psiquiátricos menores foi de $17,8 \%$ entre as entrevistadas. No que se refere à presença de sintomas musculoesqueléticos no último ano, verificou-se que a maioria dos relatos referia a desconforto e/ou dor nas regiões da coluna lombar $(75,7 \%)$, pescoço $(62,6 \%)$, coluna torácica $(57,6 \%)$ e ombros $(56,8 \%)$. Entre os que relataram sentir dor no último ano, apresentaram dor nos últimos sete 
dias $61,1 \%, 52,4 \%, 46,9 \%$ e $38,6 \%$, respectivamente para a região lombar, ombro, coluna torácica e pescoço (Tabela 3 ).

No último ano, afastaram-se do trabalho por motivo de doença/tratamento médico $32,4 \%$ das professoras. Os principais motivos de afastamento foram: gripes e resfriados $(25,0 \%)$, problemas respiratórios $(22,2 \%)$ e cirurgias gerais $(11,1 \%)$.

\section{Discussão}

No histórico pedagógico da criança, a pré-escola é o alicerce para a aprendizagem formal, é o local que a acolhe na escola e que procura garantir aos pais confiança para a primeira separação com os filhos. Comandante de toda essa responsabilidade encontra-se o professor. Este deverá ter um domínio dos conhecimentos científicos básicos necessários à aprendizagem e aos cuidados da criança (conhecimentos de saúde, higiene, psicologia, linguagem, brinquedo, expressão humana, de desenvolvimento físico entre outros). Precisa constantemente adaptar-se às situações e condições diárias de trabalho para um andamento satisfatório de suas atividades laborais.

O presente estudo trata de uma pesquisa inédita sobre saúde e condições de trabalho de professores pré-escolares da rede pública de ensino em Pelotas (RS). A maioria dos estudos existentes no Brasil e exterior descrevem e relacionam saúde e ambiente de trabalho aos professores que atuam nos ensinos fundamental, médio e universitário. Esses apresentam características diferentes da pré-escola, dificultando a validade externa dos resultados ${ }^{17,18}$.

Tabela 3. Frequência absoluta (n) e relativa (\%) das variáveis comportamentais e de saúde das professoras pré-escolares das escolas públicas da cidade de Pelotas (RS), 2010.

\begin{tabular}{|c|c|c|c|c|}
\hline Variáveis & \multicolumn{2}{|l|}{$\mathrm{N}$} & \multicolumn{2}{|l|}{$\%$} \\
\hline \multicolumn{5}{|l|}{ Autopercepção da saúde } \\
\hline Excelente & \multicolumn{2}{|l|}{5} & \multicolumn{2}{|l|}{4,5} \\
\hline Muito boa & \multicolumn{2}{|l|}{36} & \multicolumn{2}{|l|}{32,4} \\
\hline Boa & \multicolumn{2}{|l|}{49} & \multicolumn{2}{|l|}{44,2} \\
\hline Regular & \multicolumn{2}{|l|}{19} & \multicolumn{2}{|l|}{17,1} \\
\hline Ruim & \multicolumn{2}{|l|}{2} & \multicolumn{2}{|l|}{1,8} \\
\hline \multicolumn{5}{|l|}{ Hábito de fumo atual } \\
\hline Não & \multicolumn{2}{|l|}{97} & \multicolumn{2}{|l|}{87,4} \\
\hline Sim & \multicolumn{2}{|l|}{14} & \multicolumn{2}{|l|}{12,6} \\
\hline \multicolumn{5}{|l|}{ Atividade física no lazer } \\
\hline Insuficientemente ativo & \multicolumn{2}{|l|}{81} & \multicolumn{2}{|l|}{73,0} \\
\hline Ativo & \multicolumn{2}{|l|}{30} & \multicolumn{2}{|l|}{27,0} \\
\hline \multicolumn{5}{|l|}{ Índice de Massa Corporal (IMC) } \\
\hline Normal & \multicolumn{2}{|l|}{49} & \multicolumn{2}{|l|}{45,0} \\
\hline Sobrepeso & \multicolumn{2}{|l|}{44} & \multicolumn{2}{|l|}{40,4} \\
\hline Obeso & \multicolumn{2}{|l|}{16} & \multicolumn{2}{|l|}{14,7} \\
\hline \multicolumn{5}{|l|}{ Transtornos psiquiátricos menores } \\
\hline Não & 83 & & 82,2 & \\
\hline Sim & 18 & & 17,8 & \\
\hline Sintomas musculoesqueléticos & Último ano & $\%$ & Últimos 7 dias * & $\%$ \\
\hline Dor pescoço & 70 & 62,6 & 27 & 38,6 \\
\hline Dor no ombro & 63 & 56,8 & 33 & 52,4 \\
\hline Dor nos cotovelos & 16 & 14,4 & 4 & 25,0 \\
\hline Pulsos e mãos & 42 & 37,8 & 13 & 30,9 \\
\hline Coluna Torácica & 64 & 57,6 & 30 & 46,9 \\
\hline Coluna lombar & 84 & 75,7 & 52 & 61,1 \\
\hline Coxas & 16 & 14,4 & 7 & 43,7 \\
\hline Pernas & 44 & 39,0 & 17 & 38,6 \\
\hline Tornozelos & 29 & 26,1 & 13 & 44,8 \\
\hline Joelhos & 43 & 39,0 & 22 & 50,0 \\
\hline
\end{tabular}

${ }^{*} \mathrm{~N}$ e \% entre aqueles que tiveram dor no último ano 
A média de idade encontrada, a situação conjugal e o número de filhos entre os professores pesquisados é semelhante ao de outros estudos realizados com professores do ensino fundamental e médio ${ }^{9,17,19}$.

No presente estudo, todo o corpo docente era do sexo feminino. Pesquisa realizada pela Unes$\mathrm{Co}^{20}$ identificou que $81,3 \%$ dos professores no Brasil eram do sexo feminino. Essa predominância de mulheres na profissão docente pode ser explicada pelo processo histórico de inserção da mulher no mercado de trabalho, onde grande parte delas ingressou no campo educacional, sendo sua atividade rotulada como uma continuidade do trabalho doméstico, passando as professoras a assumir um papel de "mãe educadora"17.

Quanto ao grau de escolaridade, a maioria das professoras pesquisadas possui nível superior completo. Este fato se deve à implementação da Lei de Diretrizes e Bases da Educação Nacional $^{21}$, que determina a formação de docentes em nível superior para atuar na educação básica.

A referida formação é realizada através de curso de licenciatura, de graduação plena, em universidades e institutos superiores de educação, podendo ser admitida, como formação mínima para o exercício do magistério na educação infantil e nas quatro primeiras séries do ensino fundamental, a oferecida em nível médio, na modalidade Normal.

No universo pesquisado, o tempo médio de trabalho como professor de pré-escola foi relativamente menor quando comparado aos estudos de Silvany Neto et al. ${ }^{9}$, Gasparini et al. ${ }^{19}$ e Reis et al. ${ }^{22}$ onde o tempo médio encontrado foi de 10,4, 11 e 16,6 anos, respectivamente.

A grande maioria das professoras lecionava na rede municipal de ensino. A LDB, no Art. $11^{\circ}$, inciso $\mathrm{V}$, determina que os municípios devem se incumbir de oferecer a educação infantil em creches e pré-escolas, e, com prioridade, o ensino fundamental, permitindo a atuação em outros níveis de ensino somente quando estiverem atendidas plenamente as necessidades de sua área de competência e com recursos acima dos percentuais mínimos vinculados pela Constituição Federal à manutenção e desenvolvimento do ensino ${ }^{21}$.

A média de horas-aula semanal encontrada, do número de professoras que realizavam suas atividades na mesma escola (manhã e tarde) e dos docentes que relataram trabalhar em outra função remunerada diferente do magistério é muito semelhante aos dados encontrados no estudo de Medeiros et al. ${ }^{23}$ com professores do ensino fundamental de Belo Horizonte. Segundo estes autores, apesar da sobrecarga psíquica de trabalho, os baixos salários forçam os professores a realizar outro tipo de atividade remunerada.

A renda per capita dos professores oscilou entre um e três salários mínimos (mediana $=\mathrm{R} \$$ $1.000,00)$, condizente com a relatada em outros estudos ${ }^{17,19,23,24}$. Entretanto, 77,4\% das professoras pertenciam à classe $\mathrm{B}$ de acordo com o Critério de Classificação Econômica Brasil $(\mathrm{ABEP})^{15}$. Apesar de apresentarem baixa renda per capita, a maioria das professoras era casada, vivendo com companheiro, o que, provavelmente, indica aumento na renda familiar e, consequentemente, no poder de compra, critério utilizado para a categorização das classes.

O percentual de professoras que enquadraramse nas categorias referentes a sobrepeso e obesidade de acordo com a classificação do IMC é condizente com os valores encontrados em estudo nacional com mulheres da mesma faixa etária ${ }^{25}$.

A frequência de fumantes atuais foi superior à encontrada em estudos com professores da região sudeste e nordeste do país ${ }^{7,22}$. Alguns fatores podem explicar tal achado. Dados do Instituto Nacional do Câncer (INCA) ${ }^{26}$ relatam que a prevalência de fumo entre as mulheres nas capitais dos estados da região sul do Brasil, em especial do Rio Grande do Sul, são superiores a de outras capitais da união. Além disso, segundo Borges et al. ${ }^{27}$, o conhecimento não é suficiente para determinar o hábito e o abandono do fumo, visto que a sensação de prazer momentâneo ao fumar pode ser um escape às situações estressantes do cotidiano.

Entre as professoras da pré-escola, a prevalência de prática suficiente de atividade física no tempo de lazer foi maior do que a verificada na população brasileira em geral ${ }^{28}$, fato que ajuda a corroborar a hipótese de que indivíduos com maior nível socioeconômico e maior escolaridade são mais ativos no lazer ${ }^{26,27}$. Apesar disso, este resultado é bem inferior ao encontrado em estudos com professores, realizados por Delcor et al. ${ }^{17}$ (38,9\% ensino pré-escolar até ensino médio) e Vedovato e Monteiro ${ }^{24}$ (56,6 dos professores do ensino fundamental e médio praticavam atividade física regularmente, sendo que neste estudo não foi indicado o domínio utilizado). Tal diferença se dá em função das diferentes formas utilizadas para medir atividade física e os pontos de corte que determinaram o desfecho da prática suficiente (150 min/sem ou mais x prática de atividade física no lazer: sim ou não).

Foram relatadas pelas entrevistadas frequências elevadas de trabalho em posições incômodas ou viciosas, em especial trabalho nas posi- 
ções agachada e em pé. Estudos de Reis et al. ${ }^{3}$ e Delcor et al. ${ }^{17}$ encontraram prevalências similares as do presente estudo no que se refere ao trabalho na posição em pé. Relato de frequência de trabalho na posição agachada não foi encontrado em outros estudos com professores. Entretanto, entre professores que trabalham com crianças de 0 a 5 anos de idade, este é um costume frequente. Estudos de Grant et al. ${ }^{29}$ comprovam a partir de observações que os professores e auxiliares que executam suas atividade com crianças pequenas ocupam aproximadamente $25 \%$ do tempo nas posições agachada, ajoelhada ou sentada no chão. Trabalho em posições inadequadas pode, também, estar relacionado ao mobiliário disponível no local de trabalho, que 40,5\% dos professores consideraram inadequado.

O número médio de alunos por turma no presente estudo encontra-se dentro do recomendado pela Unesco e pela Organização Internacional do Trabalho ${ }^{20}$, de não ultrapassar 25 alunos por turma, o que ajuda a permitir boas condições de aprendizagem aos alunos e respeito às questões laborais do professor. Para a pré-escola, em especial, a resolução $1^{\text {a }}$ 002/2009 do Conselho Municipal de Educação do município de Pelotas $^{30}$, estabelece normas para a oferta de educação infantil, que determina um professor acompanhado de um auxiliar para turmas com mais de vinte alunos. Entretanto, apesar de cumpridas as normas municipais, foi elevada a frequência de professoras que salientaram a necessidade de possuírem auxiliares em sala de aula haja vista a dificuldade do trabalho com crianças pequenas. A quantidade elevada de alunos tem influência direta na qualidade do ensino, podendo levar ao decréscimo na aprendizagem dos mesmos e a danos à saúde dos professores em consequência do aumento de demanda em suas atividades (planejamento, correção de atividades escolares; maior atenção às crianças) $)^{31}$.

Apesar de 3/4 das professoras considerarem sua saúde como sendo muito boa ou boa, a prevalência de algumas doenças crônicas não transmissíveis é preocupante. Exemplo disso, a prevalência elevada de dor nas regiões da coluna lombar, pescoço, coluna torácica e ombros, tanto no decorrer do último ano quanto na última semana. Tais prevalências são semelhantes às encontradas nos estudos de Delcor et al. ${ }^{17}$, Vedovato e Monteiro $^{24}$, Carvalho e Alexandre ${ }^{32}$, Erick e Smith $^{33}$, Ono et al. ${ }^{34}$ e Araújo et al. ${ }^{35}$. Ono et al. ${ }^{34}$ indicam que as dores musculoesqueléticas nas regiões dos ombros, pescoço e membros superiores estão associadas a uma ampla variedade de fato- res de risco os quais incluem carga física elevada, longas horas de trabalho, falta de apoio e acúmulo de cargas de trabalho. Araújo e Carvalho ${ }^{36}$, em pesquisa com professores desde a educação infantil até o ensino superior descrevem que o trabalho repetitivo, múltiplos empregos, insatisfação no desempenho das atividades, ambiente em condições inadequadas, ritmo acelerado de trabalho, desempenho das atividades sem materiais e equipamentos adequados e salas inadequadas são variáveis associadas positivamente aos problemas identificados no presente estudo.

Apesar de importante, a presença de transtornos psiquiátricos menores entre os professores da pré-escola foi inferior à encontrada em outros estudos ${ }^{17,19,24,31,36-38}$. Vários estudos ${ }^{8,17,18,29}$ descrevem que os transtornos psiquiátricos menores ocupam o primeiro lugar entre os diagnósticos que provocaram o afastamento de professores, do ensino fundamental e médio, das suas tarefas laborais. De acordo com a literatura vigente $^{17,19,36,37,39}$ as transformações na organização do trabalho docente com novas exigências e as competências requeridas, além do volume de trabalho (50,5\% consideraram as pausas para descanso insuficientes); precariedades das condições existentes $(50,4 \%$ relataram falta de materiais para o trabalho), destacando: (37,5\%) materiais para educação artística, (28,6\%) brinquedos, (25,0\%) folhas de ofício; questões salariais, diversidade e complexidade das questões presentes na sala de aula e a expectativa social de excelência, falta de valorização (aproximadamente 20,0\% relataram não se sentir valorizadas pela direção e por colegas de outras séries com o trabalho realizado na pré-escola) podem ser, em parte, a explicação para os índices de transtornos psiquiátricos menores encontrados em professores.

Alguns aspectos devem ser destacados no presente estudo. Por um lado, além do cuidado metodológico e a inexistência de perdas e recusas, este é o primeiro estudo a descrever as condições de trabalho de professores exclusivamente da préescola pública no Brasil (estudo de Delcor et al. ${ }^{17}$ é o único que apresenta dados de professores do ensino privado pré-escolar até o ensino médio). Por outro lado, estudos ocupacionais de corte transversal só incluem indivíduos que sobreviveram à doença o que poderia ter afetado em algumas medidas de ocorrência. Devido a isso, não podemos deixar de considerar a possibilidade de havermos perdido informação daqueles professores que abandonaram a profissão em decorrência de alguma doença ou desgaste relacionado ao trabalho. No caso dos afastamentos, não houve 
prejuízo significante aos resultados encontrados devido ao fato de somente um professor estar afastado na época da coleta de dados.

Concluindo, encontrou-se uma população de professores jovens e exclusivamente do sexo feminino, com baixa renda mensal e alta demanda psicológica e física de trabalho. Destacou-se que a prevalência de professores com distúrbios psiquiátricos menores foi inferior à encontrada em professores de outros níveis de ensino e os problemas musculoesqueléticos relatados sugerem que as dores nas regiões dos ombros, pescoço e membros superiores podem estar relacionadas a uma ampla variedade de fatores de risco os quais incluem carga física elevada, falta de apoio e acúmulo de cargas de trabalho. Sugere-se a realização de novos estudos nessa população com a finalidade de investigar a associação entre as cargas de trabalho e determinados agravos à saúde.

Os resultados obtidos demonstram uma realidade, até então, pouco estudada no cenário nacional e que sugere a importância da atenção a ser dada a esse tipo de profissional ainda pouco lembrado dentro do contexto escolar. Além disso, tais achados são importantes para a criação de diretrizes e leis que ajudem a preservar as condições de trabalho e saúde desse grupo específico de docentes.

\section{Colaboradores}

LG Silva e MC Silva participaram igualmente de todas as etapas de elaboração do artigo.

\section{Referências}

1. Brasil. Lei no 9.394, de 20 de dezembro de 1996. Estabelece as diretrizes e bases da educação nacional. Diário Oficial da União 1996; 23 dez.

2. Brasil. Projeto de Lei no $144 / 2005$, aprovado pelo Senado em 25 de janeiro de 2006. Altera a redação dos arts. 29, 30, 32 e 87 da Lei no 9.394, de 20 de dezembro de 1996, que estabelece as diretrizes e bases da educação nacional, dispondo sobre a duração de 9 (nove) anos para o ensino fundamental, com matrícula obrigatória a partir dos 6 (seis) anos de idade. Diário Oficial da União 2006; 7 fev.

3. Reis EJFB, Araújo TM, Carvalho FM, Barbalho L, Silva MO. Docência e exaustão emocional. Educ Soc 2006; 27(94):229-253.

4. Mendes MLM. Condições de trabalho e saúde docente. Rio de Janeiro: Universidade Estadual do Rio de Janeiro; 2006.

5. Chong EY, Chan AH. Subjective health complaints of teachers from primary and secondary schools in Hong Kong. Int J Occup Saf Ergon 2010; 16(1):23-39.

6. Paschoal JD, Machado MCG. A história da educação infantil no Brasil: avanços, retrocessos e desafios dessa modalidade educacional. Revista HISTEDBR on line 2009; 33:78-95.

7. Araújo TM, Godinho TM, Reis EJFB, Almeida MMG. Diferenciais de gênero no trabalho docente e repercussões sobre a saúde. Cien Saude Colet 2006; 11(4):117-129.

8. Codo W. Educação: carinho e trabalho. 3a Edição. Petrópolis: Vozes; 1999.

9. Silvany-Neto AM, Araújo TM, Dutra FRD, Azi GR, Alves RL. Condições de trabalho e saúde de professores da rede particular de ensino de Salvador, Bahia. Rev Baiana Saude Publica 2000; 24(1/2):45-56.

10. Haskell WL, Lee I, Pate RR, Powell KE, Blair SN, Franklin BA, Macera CA, Heath GW, Thompson PD, Bauman A. Physical Activity and Public Health: Updated Recommendation for Adults From the American College of Sports Medicine and the American Heart Association. Circulation 2007; 116(9):1081-1093. 
11. Harding TW, Arango MV, Baltazar J, Climent CE, Ibrahim HHA, Ignacio LL, Murthy RS, Wig NN. Mental Disorders in primary health care: a study of their frequency and diagnosis in four development countries. Psychol Med 1980; 10(2):231-241.

12. Mari J, Williams PA. Validity study of a Psychiatric Screening Questionnaire (SRQ-20) in Primary Care in the city of São Paulo. Br J Psychiatry 1986; 148:2326.

13. Kuorinka I, Johnsson B, Kilbom A, Vinterberg H, Biering-Sørensen F, Andersson G, Jørgensen K. Standardized Nordic Questionnaires for the Analysis of Musculoskeletal Symptoms. Appl Ergon 1987; 18(3): 233-237.

14. Barros ENC, Alexandre NMC. Cross-cultural adaptation of the Nordic musculoskeletal questionnaire. Int Nurs Rev 2003; 50(2):101-108.

15. Associação Brasileira de Empresas de Pesquisa (ABEP). Critério de classificação econômica Brasil. [acessado 2011 nov 20]. Disponível em: http://www. abep.org/codigosguias/CCEB2008-Base2006e 2007.pdf

16. World Health Organization (WHO). Physical Status: The Use and Interpretation of Anthropometry. Geneva: WHO; 1995.

17. Delcor NS, Araújo TM, Reis EJFB, Porto LA, Carvalho FM, Silva MO, Barbalho L, de Andrade JM. Condições de trabalho e saúde dos professores da rede particular de ensino de Vitória da Conquista, Bahia, Brasil. Cad Saude Publica 2004; 20(1):187-196.

18. Gasparini SM, Barreto SM, Assunção AA. O professor, as condições de trabalho e os efeitos sobre sua saúde. Educação e Pesquisa 2005; 31(2):189-199.

19. Gasparini SM, Barreto SM, Assunção AA. Prevalência de transtornos mentais comuns em professores da rede municipal de Belo Horizonte, Minas Gerais, Brasil. Cad Saude Publica 2006; 22(12):2679-2691.

20. Organização das Nações Unidas para a educação a ciência e a cultura (Unesco). Organização Internacional do Trabalho (OIT). Compreensão e utilização da Recomendação da OIT/UNESCO de 1966 relativa ao Estatuto dos Professores e da Recomendação de 1997 da UNESCO relativa ao Estatuto do Pessoal do Ensino Superior. Geneva: UNESCO, OIT; 2008.

21. Brasil. Lei de Diretrizes e Bases da Educação Nacional Lei no 9.394. Diário Oficial da União; 1996.

22. Reis EJFB, Carvalho FM, Araújo TM, Porto LA, Silvany Neto AM. Trabalho e distúrbios psíquicos em professores da rede municipal de Vitória da Conquista, Bahia, Brasil. Cad Saude Publica 2005; 21(5):1480-1490.

23. Medeiros AM, Barreto SM, Assunção AA. Voice disorders (dysphonia) in public school female teachers working in Belo Horizonte: prevalence and associated factors. J Voice 2008; 22(6):676-687.

24. Vedovato TG, Monteiro MI. Perfil sociodemográfico e condições de saúde e trabalho dos professores de nove escolas estaduais paulistas. Rev EsC Enferm USP 2008; 42(2):290-297.

25. Brasil. Ministério da Saúde (MS). Pesquisa nacional de demografia e saúde da criança e da mulher. [acessado 2011 fev 12]. Disponível em: http://bvsms. saude.gov.br/bvs/publicacoes/pnds_crianca_ mulher.pdf
26. Ministério da Saúde (MS). Instituto Nacional de Câncer. Inquérito domiciliar sobre comportamento de risco e morbidade referida de doenças e agravos não transmissíveis: Brasil, 15 capitais e Distrito federal, 2002-2003. Rio de Janeiro; 2004.

27. Borges I, Silva Junior OC, Rego NGC. Enfermeiros professores universitários fumantes: quando o saber científico não é suficiente. Rev de Pesq: cuidado é fundamental. 2004; 8(1/2):59-69.

28. Florindo AA, Guimarães VV, Cesar CLG, Barros MBA, Alves MCGP, Goldbaum M. Epidemiology of leisure, transportation, occupational and household physical activity: prevalence and associated factors. J Phys Act Health 2009; 6(5):625-632

29. Grant KA, Habes DJ, Tepper AL. Work activities and musculoskeletal complaints among preschool workers. Appl Ergon 1995; 26(6):405-410.

30. Município de Pelotas. Resolução $1^{\text {a }} 2 / 2009$ do Conselho Municipal de Educação do município de Pelotas. Estabelece normas para a autorização de ampliação da Educação Básica em estabelecimentos de ensino da rede municipal. Pelotas: Conselho Municipal de Educação; 2009.

31. Jardim R, Barreto SM, Assunção AA. Condições de trabalho, qualidade de vida e disfonia entre docentes. Cad Saude Publica 2007; 23(10):2439-2461.

32. Carvalho AJFP, Alexandre NMC. Sintomas osteomusculares em professores do ensino fundamental. Rev Bras Fisioter 2006; 10(1):35-41.

33. Erick PN, Smith DR. A systematic review of musculoskeletal disorders among school teachers. $B M C$ Musculoskeletal Disorders 2011; 12:260.

34. Ono Y, Imaeda T, Shimaoka M, Hiruta S, Hattori Y, Ando S, Hori F, Tatsumi A. Associations of length of employment and working conditions with neck, shoulder and arm pain among nursery school teachers. Ind Health 2002; 40(2):149-158.

35. Araújo TM, Godinho TM, Reis EJFB, Almeida MMG. Diferenciais de gênero no trabalho docente e repercussões sobre a saúde. Cien Saude Colet 2006; 11(4):1117-1129.

36. Araújo TM, Carvalho FM. Condições de trabalho docente e saúde na Bahia: estudos epidemiológicos. Educ Soc 2009; 30(107):427-449.

37. Bauer J, Unterbrink T, Hack A, Pfeifer R, BuhlGriesshaber V, Müller U, Wesche H, Frommhold M, Seibt R, Scheuch K, Wirsching M. Working conditions, adverse events and mental health problems in a sample of 949 German teachers. Int Arch Occup Environ Health 2007; 80(5):442-449.

38. Chan DW. Hardiness and its role in the stress burnout relationship among prospective Chinese teachers in Hong Kong. Teaching and Teacher Education $2003 ; 19(4): 381-395$.

39. Siqueira FC, Nahas MV, Facchini LA, Piccini RX, Tomasi E, Thume E, Silveira DS, Hallal PC. Atividade física em profissionais de saúde do Sul e Nordeste do Brasil. Cad Saude Publica 2009; 25(9):1917-1928.

Artigo apresentado em 13/07/2012

Aprovado em 25/10/2012

Versão final apresentada em 12/11/2012 